\title{
Student laboratory experiments on erbium-doped fiber amplifiers and lasers
}

\section{W. Johnstone, Brian Culshaw, Douglas Walsh, David Moodie, Iain Mauchline}

W. Johnstone, Brian Culshaw, Douglas Walsh, David G. Moodie, lain S. Mauchline, "Student laboratory experiments on erbium-doped fiber amplifiers and lasers," Proc. SPIE 3831, Sixth International Conference on Education and Training in Optics and Photonics, (16 June 2000); doi: 10.1117/12.388733

Event: Education and Training in Optics and Photonics (ETOP'99), 1999, Cancun, Mexico 


\title{
Student Laboratory Experiments on Erbium Doped Fiber Amplifiers and Lasers
}

\author{
W. Johnstone ${ }^{\mathrm{a}}$, B. Culshaw ${ }^{\mathrm{a}}$, D. Walsh ${ }^{\mathrm{b}^{*}}$, D. Moodie $^{\mathrm{b}}$ and I. Mauchline ${ }^{\mathrm{b}}$

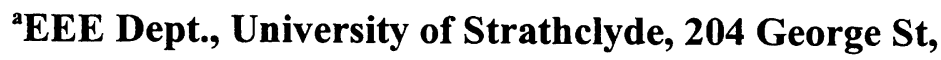 \\ Glasgow G1 1XW, Scotland UK
}

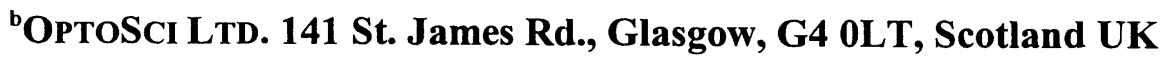

\begin{abstract}
The Erbium Doped Fiber Amplifier (EDFA) has now replaced optoelectronic repeaters as the primary design option for extending the range and capacity of the World's fiber optic telecommunications systems. In a broader sense, optical amplifiers are the basis of all lasers. It is therefore essential that students of science and engineering have a broad appreciation of, and practical familiarity with, optical amplifiers in general, EDFAs in particular and their applications in lasers. To achieve these objectives, Strathclyde University in collaboration with OPTOSCI LTD. have developed an EDFA / Laser educator kit which enables students to experimentally investigate the gain and noise characteristics of an EDFA, including issues such as signal and pump saturation, gain efficiency, amplified spontaneous emission and optical beat noise. With a simple extension to the basic amplifier kit the students are able to construct an erbium doped fiber ring laser and to investigate its power characteristics (threshold and slope efficiency) as a function of output coupling ratio and intra-cavity loss. The experimental objectives, design philosophies, hardware, experimental procedures and results will be examined in detail in this paper.
\end{abstract}

Key Words: optical amplifiers, optical communications, lasers.

\section{INTRODUCTION}

Direct optical amplification using erbium doped fiber amplifiers (EDFAs) ${ }^{1,2}$ is now preferred over optoelectronic repeaters as the primary means of restoring the signal power in long distance fiber optic links and branched networks. These amplifiers naturally provide gain at very high bit rates and at many wavelengths in a broad band stretching from $1530 \mathrm{~nm}$ to $1580 \mathrm{~nm}$. Without them high data rate wavelength division multiplexed communications systems would not exist. They also enable the implementation of multi-branch optical networks by very conveniently overcoming the large splitting losses of a high order multi-layer system. In addition, lasers (essentially optical oscillators) are simply optical amplifiers with positive feedback, again highlighting the importance of optical amplifiers in modern photonics systems. Engineering and science students therefore benefit from a good understanding and practical working knowledge of optical amplifiers and lasers. Here we report the design, hardware, experimental procedures and results of a student laboratory kit which enable the experimental investigation of erbium doped fiber amplifiers and ring lasers whilst simultaneously allowing the student to build up a broad appreciation of optical amplifiers and lasers in general.

The EDF amplifiers and lasers kit is in fact part of an integrated photonics laboratory teaching program which is centered on fiber optic communications systems to provide specific experimental experience of this field as well as broad exposure to many of the most important principles of photonics in general. This program, reported in full elsewhere ${ }^{3}$, utilizes four custom designed kits of photonics hardware which enable comprehensive experimental investigations on optical waveguides, a $1 \mathrm{~km}$ point to point fiber optic communications link, the EDF kit reported here and a fiber optic network analyzed by a standard test instrument (an optical time domain reflectometer - OTDR). With these four experimental systems we cover most of the essential elements of photonics courses addressing the principles, characteristics and applications of optical waveguides, optical fibers, optical amplifiers, lasers (in particular semiconductor lasers), light

\footnotetext{
" Correspondence: Tel: +44 (0) 141552 7020; Fax: +44 (0) 141552 3886; Email: info@optosci.com; WwW: http://www.optosci.com
} 
emitting diodes (LEDs), photodiode optical detectors, optical receivers and optical communications systems. The experiments have been designed for intermediate and advanced level courses aimed at undergraduates in the upper-years of their studies or post graduates at Masters level. In addition, they may be useful for technical colleges and training institutes where students are undertaking specific vocational training courses in photonics or optical communications technologies.

When constructing a student laboratory teaching experiment, it is often inadequate to take existing equipment designed perhaps for research or engineering applications and then formulate teaching experiments around it. Success will be assured only by taking a fully integrated approach to the design of laboratory based photonics teaching packages including the design of dedicated hardware, experimental procedures, exercises and manuals. To ensure that all desirable educational objectives are met and that all of the most important scientific and technical principles, issues and phenomena are addressed, we have developed our suite of four fully integrated laboratory based teaching packages in accordance with the following design rules:

- Define the educational objectives in terms of the physical principles, important technical features, design issues and performance characteristics which must be addressed, with particular attention to facilitating student understanding and ability to implement concepts.

- Define the experiments to meet these performance objectives.

- Design the dedicated (custom) hardware to enable the proposed experimental investigation whilst keeping costs within realistic academic teaching budgets.

- Formulate the experimental procedure and manuals to guide the students through the investigation and results analysis (in some cases more open ended investigations may be formulated with minimal guidance to the students).

- Formulate tutorial exercises and case studies to relate the results to real world devices and systems.

The primary constraint is cost and the final packages must be affordable within higher education budgets. In general, the packages have been designed as far as possible to be self contained in that as little ancillary equipment as possible is required. However, where it is advantageous and cost effective to use equipment normally available in student laboratories, the packages have been designed to be compatible with the capabilities of such equipment e.g. 20 or $50 \mathrm{MHz}$ oscilloscopes and waveform generators.

The four packages now fully developed on the basis of the above approach cover the following topics: Principles of Optical Waveguides. Fiber Optic Communications Systems, Erbium Doped Fiber Amplifiers and Lasers and Optical Network Analysis. Here, we present outline descriptions of the educational objectives, the hardware and the experimental procedures of the EDF amplifiers and lasers experimental teaching package.

\section{THE EDF AMPLIFIER AND LASER EXPERIMENT}

The objectives of the EDF optical amplifiers and lasers experiment are to enable students to experimentally investigate the principles and characteristics of erbium doped fiber amplifiers and lasers. The underlying aims are to allow them to gain practical, hands experience of EDF amplifiers and lasers in particular and to consolidate their conceptual understanding and knowledge of optical amplifiers and lasers in general.

To achieve the above aims and objectives the experimental investigation was defined as follows:

- measurement and analysis of gain as a function of signal power and pump power including determination of the point of transparency, the gain efficiency and the gain gradient,

- investigation and analysis of small and large signal gain,

- investigation of gain saturation,

- investigation of pump saturation,

- determination of saturated output power as a function of pump power,

- investigation of amplified spontaneous emission (ASE) and ASE noise and its dependence on pump and signal power,

- investigation of Signal-ASE and ASE beat noise and their influence on the amplifier noise figure,

- construction of an EDF laser and investigation of its output characteristic (threshold and slope efficiency) as a function of the output coupling ratio and the intra-cavity loss. 
The equipment designed specifically to enable the above experimental program comprises two units: the EDFA and a Signal Source and Receiver Unit which is used to provide input signal power to the amplifier and to measure the output power. Erbium doped fiber (EDF) is a 3 level optical gain medium providing amplification by stimulated emission at $1550 \mathrm{~nm}$ when pumped at $980 \mathrm{~nm}$. The complete amplifier package including the EDF is of a fairly standard design ${ }^{1,2}$ (see Figure 1) pumped by a $70 \mathrm{~mW} 980 \mathrm{~nm}$ diode laser via a $980 / 1550 \mathrm{~nm}$ wavelength division multiplexer (WDM). It is capable of gains in excess of $20 \mathrm{~dB}$ and saturated output powers in the range of $20-25 \mathrm{~mW}$ making it useful as a stand alone amplifier in real applications. The pump power, and hence the gain of the amplifier, is varied by adjusting the pump diode bias current. Residual pump power is measured by the monitor photodiode at the redundant arm of the WDM and, with appropriate calibration, the actual pump power is displayed on the front panel in $\mathrm{mW}$. An optical isolator and angle polished connectors eliminate problems with optical feedback and any possibility of oscillation. The WDM at the output of the erbium doped fiber dumps any residual pump power preventing significant levels of potentially eye damaging $980 \mathrm{~nm}$ radiation from reaching the output connector.

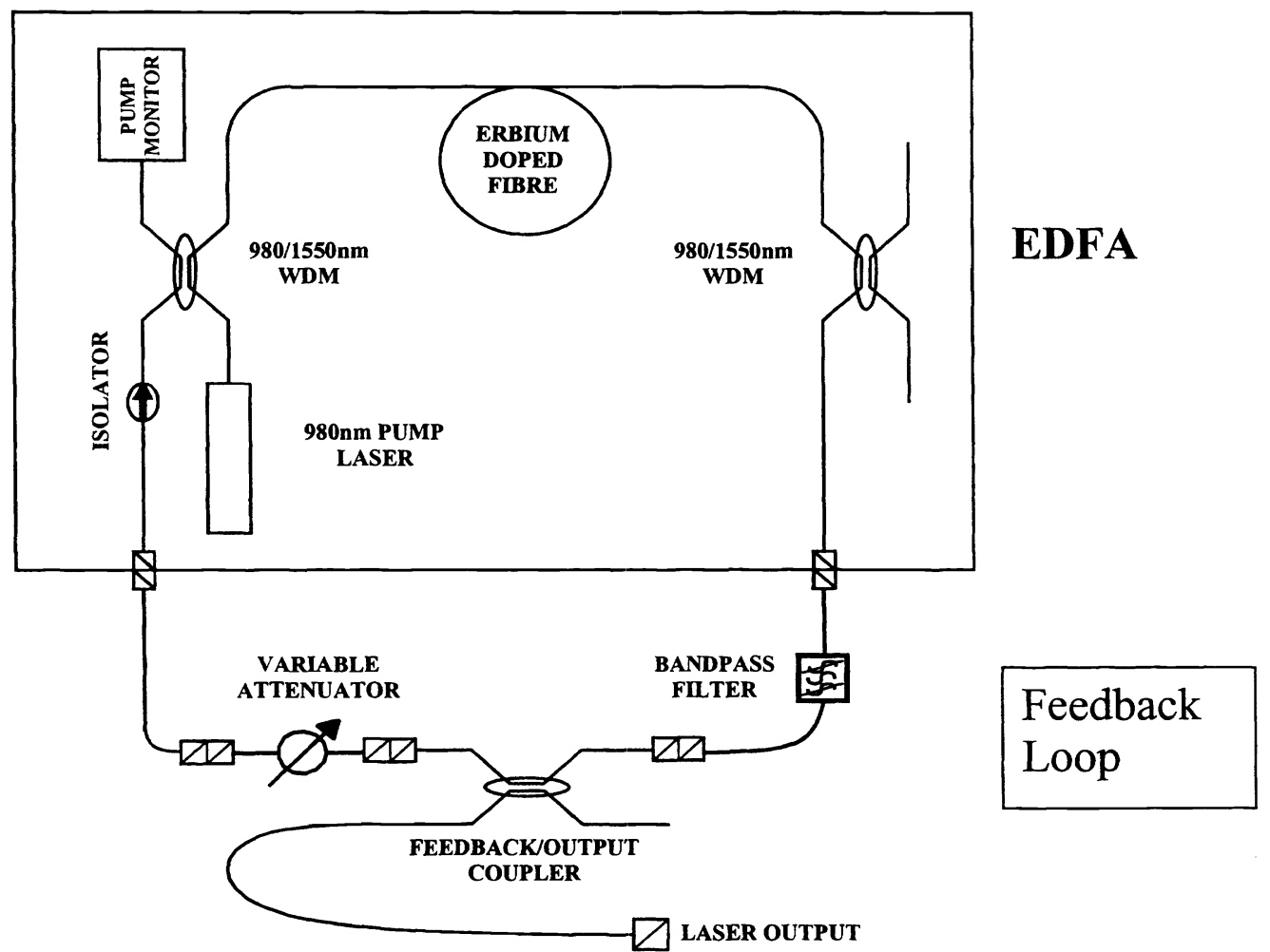

Figure 1: Schematic diagram of EDF amplifier with the feedback loop to create a fiber ring laser

The Signal Source and Receiver Unit is a two part instrument consisting of a Signal Laser module which provides the input signal for the experiments on the EDFA and a Photoreceiver module which measures the powers of the input / output signals used. The signal source is a single frequency DFB laser and it may be operated with constant output power or modulated with a $100 \mathrm{kHz}$ sinusoid. A DFB laser is necessary to eliminate mode partition noise which masks the SignalASE and ASE-ASE beat noise contributions. The signal power delivered to the amplifier input may be varied in a $45 \mathrm{~dB}$ dynamic range $(0 \mathrm{dBm}[1 \mathrm{~mW}]$ to $-45 \mathrm{dBm}[30 \mathrm{nW}]$ approximately) by adjusting a variable optical fiber attenuator mounted in the source unit. This enables small and large signal gain conditions to be investigated without any change in the wavelength characteristics of the source.

The Photoreceiver module uses a standard InGaAs photodiode with a $100 \mathrm{kHz}$ lock-in amplifier (LIA) detection scheme which may be engaged or not as required. A signal frequency of $100 \mathrm{kHz}$ is significantly greater than the relaxation frequency of the amplifier and hence, the amplified spontaneous emission (ASE) and the population inversion is not modulated significantly in response to the signal. With the laser modulation and LIA detection scheme engaged, the receiver unit thus measures only the modulated amplified signal power, rejecting the $\mathrm{CW}$ amplified spontaneous emission. 
In Average mode, the total averaged incident power including constant power components such as the ASE contribution is measured. To enable investigation of the signals using an oscilloscope or spectrum analyzer the direct signal from the photodiode output is available at the BNC on the front panel. These features provide the flexibility required to measure AC signal power only and separate out constant power levels from the amplified spontaneous emission.

Inserting a fused fiber coupler from the EDFA output back to the input provides the necessary feedback for laser operation with the output emerging from one of the spare arms of the coupler (Figure 1). The isolator in the amplifier ensures unidirectional oscillation and a band pass filter inserted before the output coupler defines the operating wavelength and prevents ASE from reaching the output to corrupt the measurement of the laser power characteristics. Two fused fiber couplers (a 20:80 and a 40:60) are used to enable the investigation of 4 possible output coupling ratios. All are fitted with angle polished connectors to minimize parasitic feedback and oscillation. In addition, a fiber optic variable attenuator can be inserted into the feedback loop to allow a study of the effects of intra-cavity loss.

\section{RESULTS AND ANALYSIS}

In the lectures and in the notes accompanying the experiments we develop a plane wave model of an optical amplifier ${ }^{4}$ with a three level gain medium arriving at an expression for the small signal gain, $G_{0}(v)$, of an amplifier of length $l$ :

$$
G_{0}(v)=\exp \left[\gamma_{0}(v) l\right]
$$

where $\gamma_{0}(v)$ is the small signal gain coefficient.

It is customary to express the gain of an amplifier in $\mathrm{dB}$ and from equation 1 we get:

$$
\operatorname{Gain}(d B)=10 \log _{10} G_{0}(v)=10 \log _{10} e^{\eta t}=4.34 \gamma(v) l
$$

As we increase the pump power, the population of the upper gain state increases linearly as does the population inversion and the gain coefficient. At very low pump powers the population inversion is insufficient to provide gain and the signal is attenuated (by an amount depending on the population of the lower gain state). As the pump power, the population inversion and stimulated emission increase, the attenuation decreases and the system becomes transparent. Beyond the point of transparency (gain $=0 \mathrm{~dB}$ ), the gain (in $\mathrm{dB}$ ) increases linearly with $\gamma(v)$ in accordance with equation 2 and hence increases linearly with pump power with pump power.

It must be noted that the above model only applies for small input signals under weak pumping conditions for which we can assume insignificant depletion of the ground state. From a detailed analysis ${ }^{4}$ of a 3 level gain medium, such as erbium doped glass, in which weak signals and weak pumping are not assumed we get an expression for the gain coefficient, $\gamma(v)$ at a signal intensity level of $\mathrm{I}_{v}$, as follows:

$$
\gamma(v)=\frac{1}{I_{v}} \cdot \frac{d I_{v}}{d z}=\frac{\left(I_{p}^{*}-1\right) \sigma_{S E} N_{t}}{1+2 I_{v}^{*}+I_{p}^{*}}
$$

where $\mathrm{N}_{\mathrm{t}}$ is the atomic density of the medium, $\sigma_{\mathrm{SE}}$ is the stimulated emission cross-section, $I_{v}^{*}=I_{v} / I_{s}$ and $I_{p}^{*}=I_{p} / I_{p s}\left(\mathrm{I}_{\mathrm{p}}\right.$ is the pump intensity and $\mathrm{I}_{\mathrm{ps}}$ and $\mathrm{I}_{\mathrm{s}}$ are constants referred to as the pump saturation intensity and signal saturation intensity respectively).

For a fixed pump power, equation 3 indicates that, as the signal intensity increases above $I_{s}$, the term $2 I_{v}{ }_{v}^{*}$ in the denominator increases and the gain falls off as the system experiences the phenomenon referred to as gain saturation. This arises from significant depletion of the upper gain state population by the high rate of stimulated emission. The saturated output power of the amplifier is that for which the gain has fallen by $3 \mathrm{~dB}$ with respect to the small signal gain. In addition, for a fixed input signal intensity, equation 3 indicates that the gain coefficient and hence the overall gain in $\mathrm{dB}$ (see equation 2 ) initially increases linearly with pump intensity, $I_{p}$, but then flattens out as $I_{p}$ approaches and exceeds $I_{p s}$, increasing $I_{p}^{*}$ in the 
denominator. Intuitively, such behavior is expected (and observed experimentally, see results discussed below) since a large pump power will result in severe depletion of the ground state leading to reduced absorption and pumping rate. This phenomenon, referred to as pump saturation, prevails in all operating 3 level systems where the lower gain state is the ground state which by definition must be at least $50 \%$ depleted simply to obtain a population inversion. It is also evident in 4 level systems under strong pumping conditions.

By varying the current to the pump laser and the level of in-line attenuation at the signal laser output the students study the gain characteristics of the EDFA as a function of pump power and input signal power. Figure 2 shows sample results for the variation of gain with input signal power at several levels of pump power. The students can clearly see and report on the effects of gain saturation as the signal level becomes large enough to significantly deplete the population inversion and the amplifier gain falls off in accordance with equation 3.

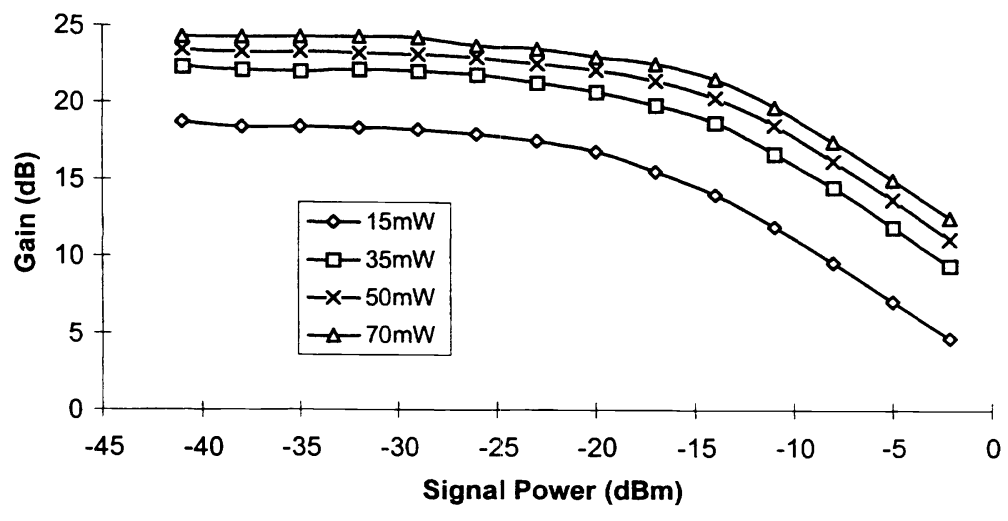

Figure 2: Gain versus signal power for various pump powers

Figure 3 shows the variation of gain with pump power at several levels of signal input power. Here the students can clearly observe and report that initially the gain (in $\mathrm{dB}$ ) increases linearly with pump power (equation 2) until the effects of pump saturation become evident as the high level of pump light significantly depletes the population of the ground state (equation 3). They can also determine the point at which the amplifier is transparent (i.e. gain =1), the gain gradient and gain efficiency (see Figure 3). In addition, they carry out exercises on saturated output and input power which can be measured from these curves and relate their findings to the conceptual understanding gained from the model presented in the notes and briefly annotated above.

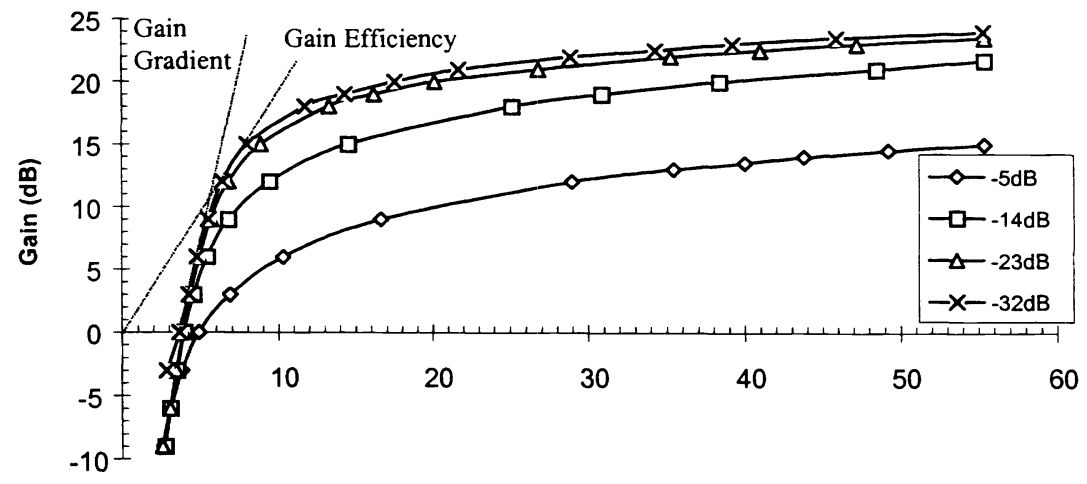

Pump Power (mW)

Figure 3: Gain versus pump power for several input signal levels (Gain Gradient $=5.56 \mathrm{~dB} / \mathrm{mW}$, Gain Efficiency $=2 \mathrm{~dB} / \mathrm{mW}$ )

Noise associated with amplified spontaneous emission (ASE) ${ }^{1}$ is the limiting factor in determining the ultimate signal to noise ratio in any system using optical amplifiers. Figure 4 shows the variation of the level of ASE with increasing signal 
power at several levels of pump power, as measured by the students. ASE is responsible for degradation of the signal to noise ratio in systems using optical amplifiers by contributing ASE-ASE beat noise and Signal ASE beat noise. In Figure 4 the students can clearly see that the ASE levels fall dramatically as the input signal level increases into the gain saturation region and the population inversion of the amplifier falls with a corresponding decrease in spontaneous emission and gain. Further studies also show the decrease in optical noise levels (signal-ASE and ASE-ASE beat noise) as we go into the region of signal saturation. Again simple models of the ASE generation and the beat noise terms enables the students to relate their findings on ASE level changes and optical noise variations to the basic physical processes of the gain medium. Further exercises also illustrate the impact of noise and the amplifier operating conditions on the performance of optical communications systems.

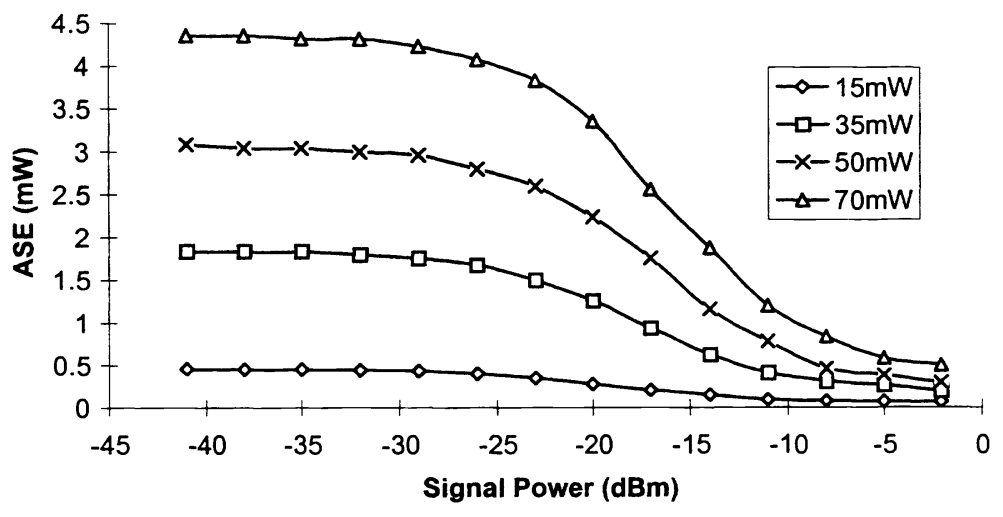

Figure 4: ASE power versus signal power for several values of pump power

Following a full characterization of the amplifier including a complete study of the gain and noise characteristics, the students use the fused fiber feedback couplers, the filter and the variable attenuator (as shown in Figure 1) to construct and then characterize an erbium doped fiber ring laser ${ }^{2}$. A simple model of a 3 level ring laser ${ }^{4}$ is developed in the accompanying notes resulting in an expression for the output power, $\mathrm{P}_{\mathrm{o}}$, as a function of the small signal gain coefficient, with intra-cavity loss $\left(\mathrm{L}_{\mathrm{i}}\right)$ and output coupling ratio $\left(\mathrm{T}_{\mathrm{o}}\right)$ as variables:

$$
P_{0}=\frac{T_{o} P_{s} \gamma_{o}(v) l}{1-L_{i}\left(1-T_{o}\right)}+\frac{\ln \left[L_{i}\left(1-T_{o}\right)\right] T_{0} P_{s}}{1-L_{i}\left(1-T_{o}\right)}
$$

where $P_{S}$ is the saturation power.

Since $\gamma_{0}(v) . l$ is proportional to the pump power, $\mathbf{P}_{\mathrm{p}}$, and letting $C$ be the proportionality constant we can write the output power in terms of the pump power as:

$$
P_{0}=\left(\frac{T_{o} P_{s} C}{1-L_{i}\left(1-T_{o}\right)}\right) \cdot P_{p}+\frac{\ln \left[L_{i}\left(1-T_{o}\right)\right] T_{0} P_{s}}{1-L_{i}\left(1-T_{o}\right)}
$$

This is the equation of a straight line intersecting the pump power axis at the laser threshold point determined by the intracavity loss, $L_{i}$, the output coupling ratio, $T_{0}$ and the saturation power, $P_{s}$. The threshold is the point at which the pump power provides a small signal gain which exactly offsets the total intrinsic loss around the cavity, $\mathrm{L}_{\mathrm{i}}\left(1-\mathrm{T}_{0}\right)$. The gradient of the line, known as the slope efficiency (SE) of the laser is given by:

$$
S E=\frac{T_{0} P_{s} C}{1-L_{i}\left(1-T_{0}\right)}
$$


Since the EDF laser is identical in principle to that analyzed above all of the equations presented here apply in a relative sense. The students measure the output power characteristics as a function of the output coupling ratio and the intra-cavity loss. Figure 5 shows one example of the variation in the laser output characteristics with output coupling ratio for a fixed internal excess loss of $13 \mathrm{~dB}$. Clearly the threshold and the slope efficiency increases with output coupling ratio. For a high loss cavity, the denominator of equation 6 increases only very weakly with $T_{o}$ and the slope efficiency is expected to increase approximately linearly with $\mathrm{T}_{\mathrm{o}}$. A full analysis of Figure 5 confirms this experimentally and shows that the threshold gain increases exactly to off-set the increasing loss.

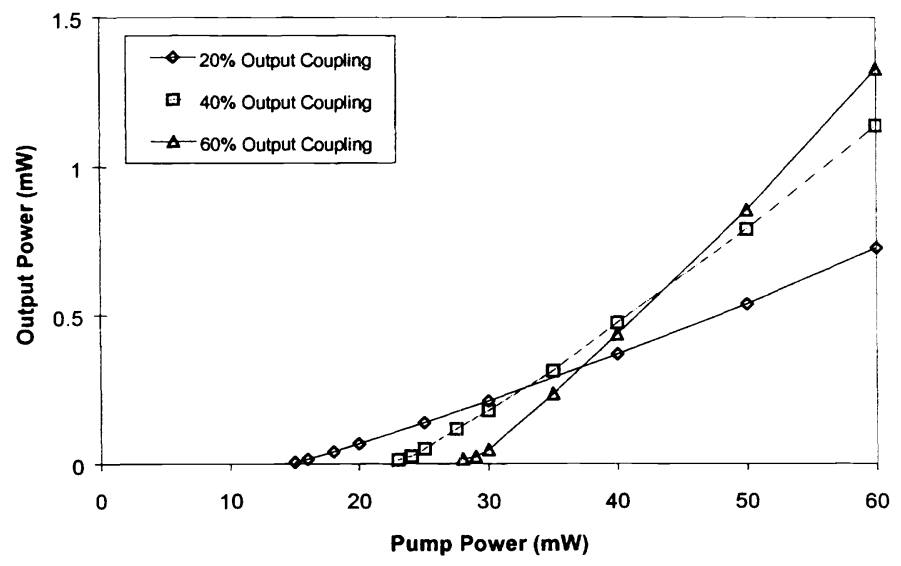

Figure 5: Output power vs. pump power for various output coupling ratios at -13dB intra-cavity loss

Figure 6 shows an example of the variation of the laser output characteristic with excess intra-cavity loss for an output coupling ratio of $60 \%$. For a high output coupling ratio and high loss, the denominator of equation 6 varies only weakly with $\mathrm{L}_{\mathrm{I}}$, predicting a weak variation of slope efficiency. This is again confirmed by the results presented in Figure 6 and a full analysis indicates again that the threshold gain increases exactly to off-set the increasing loss as expected (Figure 7).

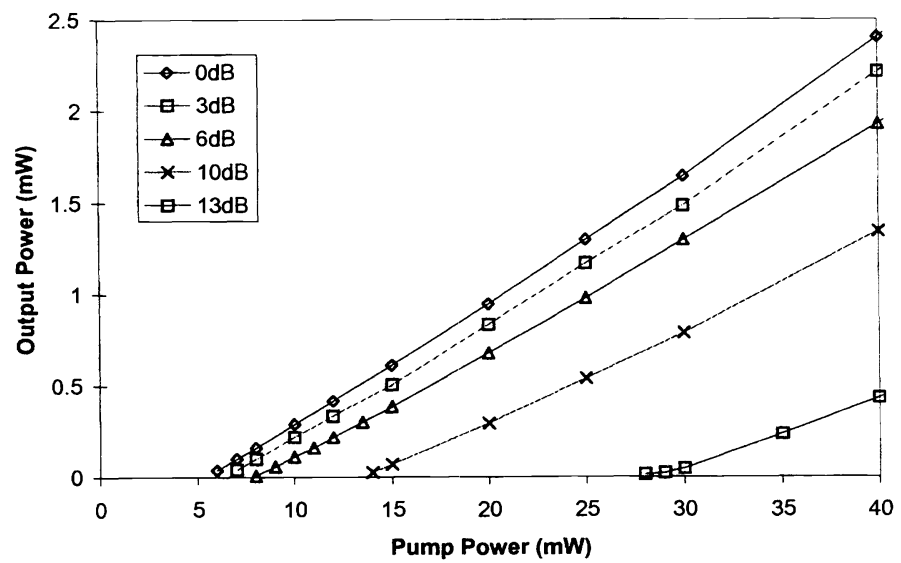

Figure 6: Output power vs. pump power for the various levels of intra cavity loss for an output coupling ratio of $60 \%$ 


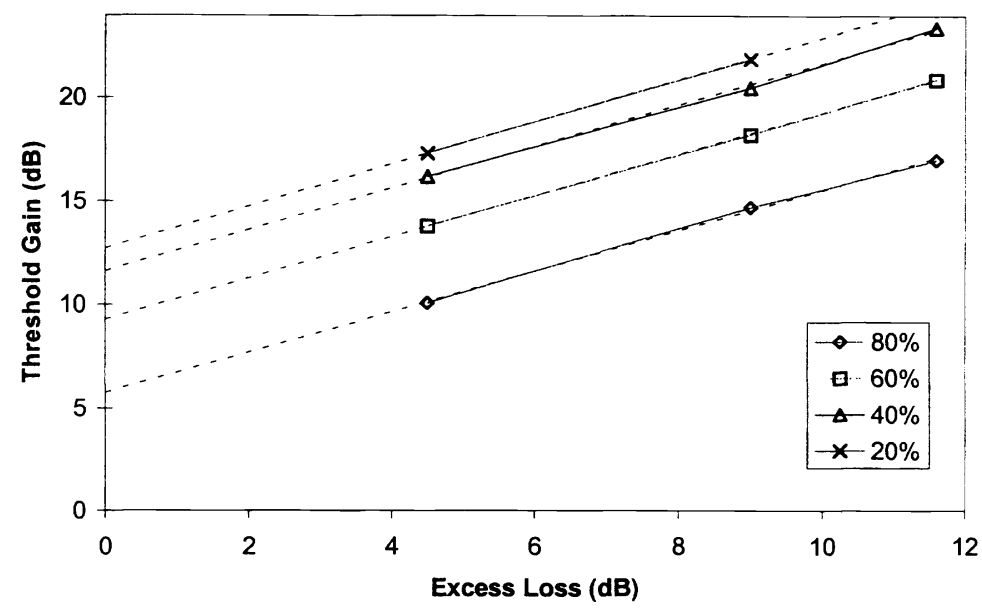

Figure 7: Threshold gain versus excess intra-cavity loss for various output coupling ratios

\section{CONCLUSIONS}

An experimental teaching package on EDF amplifiers and lasers has been developed for modern optics, photonics and optical communications courses. It is suitable for both physics and engineering based courses since it addresses fundamental physical principles, key technical issues, component and system performance characteristics and design issues. The educational objectives were firstly defined and the necessary custom hardware and experimental procedures were then designed to achieve these objectives. This approach has ensured that all of the key physical principles and their technological implementation are addressed.

The erbium doped fiber amplifier is a complex physical system and the principal student benefit from this experiment is to appreciate that a relatively straightforward physical model can provide good insight into the operation and performance limits of a complex entity such as the EDFA or the EDF ring laser. In the student manual a simple plane wave model of a 3 level bulk optical amplifier ${ }^{4}$ predicts such features as point of transparency, gain efficiency, signal and pump saturation and ASE based noise contributions and the relationship of the noise and signal outputs to pumping rate, population inversion, upper state lifetime and input power. The students measure these features in the EDFA and are able to relate their results in a relative manner to the model presented. Hence, the experimental investigation reinforces the student understanding of the physical phenomena which determine the characteristics of EDFAs.

As a continuation to the amplifier investigation the students construct various laser cavities with the equipment described above and investigate the laser threshold and slope efficiency as a function of intra-cavity loss and output coupling ratio. A simple model of the output power characteristics of a ring laser ${ }^{4}$ is developed in the accompanying notes and the students are able to relate the relative variations in threshold and slope efficiency to the intra-cavity loss, output coupling ratio and gain of the amplifying medium. The experiment thus provides genuine insight into the performance of laser systems as a function of their most important design parameters.

The basic physics of fiber amplifiers and lasers is extremely challenging. Feedback confirms that our students gain considerable satisfaction and confidence from seeing such complex physics in action as an engineering component. The students claim that they have not only enjoyed these laboratory exercises but that they feel they have learned a great deal that is both fundamental and directly useful in the real technical world beyond their studies. This is borne out by the quality of their laboratory reports which exhibit both physical understanding and technological critique - surely the essential aim in the educational process in applied science and engineering. 


\section{REFERENCES}

1. G. P. Agrawal, Fiber Optic Communications Systems, John Wiley and Sons, 1997.

2. M. S. Digonnet, Fiber Amplifiers and Lasers, Marcell Dekker, 1994.

3. W. Johnstone, B. Culshaw, D. Moodie, I. Mauchline and D. Walsh, "Photonics laboratory experiments for modern technology based courses", IEEE Proceedings, Special issue on "Optics Education”, to be published in 1999.

4. J. Verdyne, Laser Electronics, Prentice Hall, 1985. 